\title{
Conjugate Effects of Buoyancy and Magnetic Field on Heat and Fluid Flow Pattern at Low-to-Moderate Prandtl Numbers
}

\author{
Ridha DJEBALI ${ }^{1,2, *}$, Mohamed Ammar ABBASSI ${ }^{2}$ and Ahlem ROUAHI ${ }^{3}$ \\ ${ }^{1}$ ISLAIB - Béja 9000, University of Jendouba, Tunisia. \\ ${ }^{2}$ Univ. Gafsa, RU: Materials, Energy and Renewable Energies (MEER), Tunisia \\ ${ }^{3}$ LMOP, University of Tunis El Manar, 2092 Tunis, Tunisia. \\ *jbelii_r@hotmail.fr or ridha.djebali@ipein.rnu.tn
}

Keywords: Lattice Boltzmann computer modelling, Prandtl number effect, electrically conducting fluid, heat transfer, magnetoconvection.

\begin{abstract}
This study aims to present a numerical investigation of unsteady two-dimensional natural convection of an electrically conducting fluid in a square medium under externally imposed magnetic field. A temperature gradient is applied between the two opposing side walls parallel to ydirection, while the floor and ceiling parallel to x-direction are kept adiabatic. The coupled momentum and energy equations associated with the Lorentz 'decelerating' force as well as the buoyancy force terms are solved using the single relaxation lattice Boltzmann (LB) approach. The flow is characterized by the Rayleigh number $\operatorname{Ra}\left(10^{3}-10^{6}\right)$, the Prandtl number $\operatorname{Pr}(0.01-10)$, the Hartman number $\mathrm{Ha}$ (0-100) determined by the strength of the imposed magnetic field and its tilt angle from $\mathrm{x}$-axis ranging from $0^{\circ}$ to $90^{\circ}$. The changes in the buoyant flow patterns and temperature contours due to the effects of varying the controlling parameters and associated heat transfer are examined. It was found that the developed thermal LB model gives excellent results by comparison with former experimental and numerical findings. Starting from the values $10^{5}$ of the Rayleigh number $R a$ and $H a=0$, the flow is unsteady multicellular for low Prandtl number typical of liquid metal. Increasing gradually $\mathrm{Pr}$, the flow undergoes transition to steady bicellular. The transition occurs at a threshold value between $P r=0.01$ and 0.1 . Increasing more the Prandtl number, the flow structure is distorted due to the viscous forces which outweigh the buoyancy forces and a thermal stratification is clearly established. For high Hartman number, the damping effects suppress the unsteady behaviour and results in steady state with extended unicellular pattern in the direction of Lorentz force and the heat transfer rate is reduced considerably.
\end{abstract}

\section{INTRODUCTION}

Study of magnetohydrodynamic (MHD) flows, has been the subject of large number of numerical investigations [1-3]. Moreover, flows under external magnetic field are of practical interest such as crystal growth in liquids, cooling of nuclear reactors, electronic packages, micro electronic devices; and have been the subject of many earlier and recent studies for free fluid flows [4-6]. Furthermore, the study of flow and heat transfer in electrically conducting fluid has received considerable attention and renewed interest due to the many applications in engineering problems. -

\section{Nomenclature}

\begin{tabular}{llll}
\hline $\boldsymbol{B}=$ & Magnetic field & $N u=$ & Nusselt number at the hot wall \\
$\boldsymbol{e}_{\boldsymbol{k}}=$ & Discrete lattice velocity & $R a=$ & Rayleigh number $g \beta \Delta H^{3} / v \alpha$ \\
$\boldsymbol{F}=$ & Forcing term & Greek & symbols \\
$\boldsymbol{g}=$ & Gravitational acceleration & $w_{k}=$ & Weighting factors \\
$\boldsymbol{u}=$ & Velocity vector $(u, v)$ & $\rho=$ & Fluid density \\
$\boldsymbol{x}=$ & Lattice node in $(x, y)$ coordinates & $v=$ & Kinemtic viscosity \\
$c_{s}=$ & Lattice sound speed & $\alpha=$ & Thermal diffusivity
\end{tabular}




\begin{tabular}{llll}
$f_{k}, g_{k}$ & Discrete distribution functions & $\tau_{v}, \tau_{\alpha}$ & Relaxation times for $f_{k}$ and $g_{k}$ \\
$p=$ & Ideal gas pressure $\rho c_{s}{ }^{2}$ & $\beta=$ & Thermal expansion coefficient \\
$T=$ & Temperature field & $\sigma=$ & Fluid Electrical conductivity \\
$\Delta T=$ & Temperature gradient $T_{h}-T_{c}$ & $\theta=$ & Dimensionless temperature \\
$\Delta t=$ & Time step & $\gamma=$ & Magnetic field inclination \\
$\Delta x=$ & Lattice spacing units $(=\Delta y)$ & Subscripts and Superscripts \\
$H=$ & Height of the enclosure & $e q=$ & Equilibrium part \\
$H a=$ & Hartmann number & $i, j=$ & Lattice vector components \\
$P r=$ & Prandtl number $\mathrm{v} / \alpha$ & $k=$ & Discrete velocity direction \\
\hline
\end{tabular}

- One can cite for example such as MHD generators, plasma studies, nuclear reactors, and because of the effect on magnetic fields on the performance of many systems including liquid metals and alloys, mercury amalgams, and blood, known as "low-Prandtl number flows".

Natural convection in enclosures depends strongly on many parameters monitoring the flow behaviour in special industrial situations, namely the Rayleigh and the Prandtl number, the medium tilting angle and its configuration.

Besides, several numerical simulations have been conducted in the past using conventional numerical methods based on discretization of macroscopic equations. Recently, the Lattice Boltzmann Method (LBM) has met with significant success for numerical simulation and modelling of many classical and complex flows [7-10]. The LBM has been used recently to investigate flows under external magnetic field. For citation, Hao et al. (2011) [11] simulated a multicomponent system formed by fluid and magnetic particles using a multiphase LB model, under external magnetic field. Authors concluded that the LB method is a so helpful to explore and understand the chainlike particles behaviour when applying external magnetic field on a random distribution of particles. Chatterjee and Amiroudine (2011) [12] used a non-isothermal LB model to predict the thermo-fluidic phenomena in a direct current MHD micropump. It was remarked that flow and heat transfer characteristics depend strongly on Hartmann, Prandtl and Eckert numbers and channel aspect ratios. An excellent agreement is also observed between the LB results and experimental, analytical and other available numerical results in the literature. Han (2009) [13] used the FV approach to investigate MHD natural convection flow for a tilted square cavity. It was concluded that for high magnetic field strength, the velocities are suppressed and the convective heat transfer rate is reduced and the effect of a magnetic field is found to decrease the Nusselt number considerably, regardless of the inclination angle. Ece and Büyük (2006) have investigated the steady laminar natural-convection flow in an inclined square enclosure heated and cooled from adjacent sides in the presence of a magnetic field. The governing equations based on the stream function, vorticity and temperature have been solved using the Differential Quadrature Method for various Grashof and Hartman numbers and aspect ratios, inclination of the cavity and magnetic field orientations. Its has been observed that the flow characteristics, therefore the heat transfer rate are affected significantly by the variation of Hartman number, the aspect ratio and the inclination of the enclosure. Zhang et al. (2010) [14] introduced a LB model to investigate a thermo-sensitive magnetic fluid in porous medium. Authors obtained excellent agreement with previous results and concluded that the LB method is a promising tool for understanding magnetic fluid non-isothermal behaviour in porous media.

Through this literature review, one can state that flow patterns and temperature field exhibit distinctly different behaviour in differentially heated enclosures by varying the monitoring parameters: Rayleigh number and Hartmann number (Magnetic field strength). Additionally, no or very little works has been reported on this topic with regard to the Prandtl number effects. In the present paper, a thermal lattice Boltzmann model is developed and used to investigate the dynamic and thermal behaviour in electrically conducting fluid in a square enclosure. The effects of the Rayleigh number, the Prandtl number and the magnetic field strength and its orientation -in wide ranges- on flow and heat transfer are analyzed and tabulated for benchmarking purposes. 


\section{PROBLEM STATEMENT}

The investigated problem is a two-dimensional square enclosure of edge $H(=L)$ filled with a viscous, incompressible and electrically conducting fluid. A temperature difference $\Delta \mathrm{T}=T_{h^{-}} T_{c}$ is applied between walls parallel to y-direction which is responsible of the convective onset and motion. The fluid is permeated by a uniform magnetic field of strength B tilted by an angle $\gamma$ with respect to $x$-axis (Fig. 1). The gravity field reigns in the vertical descendant direction. It is assumed that the induced magnetic field can be neglected in comparison with the imposed magnetic field.

We assume all fluid properties including the electrical conductivity to be constant, except the density which is linearly temperature-dependent, so that the Boussinesq approximation is used: $\rho=\rho_{0}\left(1-\beta\left(T-T_{\infty}\right)\right)$ where $T_{\infty}$ is the reference temperature taken here the cold temperature. Neglecting viscous heat dissipation and compression work done by the pressure, the unsteady state governing equations can be summarized as follows:

\section{Continuity}

$$
\nabla . \boldsymbol{U}=0
$$

\section{Momentum}

$$
\partial_{t} \boldsymbol{U}+(\boldsymbol{U} . \nabla) \boldsymbol{U}=-\nabla P+\nabla .(v \nabla \boldsymbol{U})+\rho \boldsymbol{F} / \rho_{0}
$$

$$
\begin{aligned}
& \text { Energy } \\
& \partial_{t} T+(\boldsymbol{U} \cdot \nabla) T=\nabla \cdot(\alpha \nabla T)
\end{aligned}
$$

Where $F_{i}=\beta\left(T-T_{\infty}\right) g \delta_{i 2}+\sigma\left[\left(B_{j} U_{j}\right) B_{i}-B^{2} U_{i}\right]$ and $B_{j}$ denotes the magnetic fields components $B_{x}=B \cos (\gamma)$ and $B_{y}=B \sin (\gamma)$. We assume the Joule heating can be neglected since $R a>10^{3}$ and $H a<200$.

For the sake of comparison with previous studies, all predicted quantities are presented in a non-dimensional form. The reference scales $l_{0}=H, u_{0}=\alpha / H t_{0}=H^{2} / \alpha, p_{0}=\rho_{0} U_{0}^{2}$ and $\Delta T=T_{h}-T_{c}$ are used for length, velocity, time pressure and relative temperature respectively. The reference temperature is chosen to be $T_{c}$.

The flow is characterized by the Rayleigh number $\left(10^{3} \leq R a \leq 10^{6}\right)$, the Prandtl number $(0.01 \leq \operatorname{Pr} \leq 10)$, the Hartmann number $(0 \leq H a \leq 100)$ and the magnetic field orientation $\left(0^{\circ} \leq \gamma \leq 90^{\circ}\right)$, defined as:

$$
R a=g \beta \Delta T H^{3} / v \alpha, P r=\frac{v}{\alpha} \text { and } H a=H B \sqrt{\sigma / \mu}
$$

The following changes of variables are implemented in the following:

$$
x=\frac{X}{l_{0}}, y=\frac{Y}{l_{0}}, u=\frac{U}{u_{0}}, v=\frac{V}{u_{0}}, p=\frac{P}{p_{0}}, \theta=\frac{T-T_{c}}{\Delta T}
$$

Based on the presumptions above, the dimensionless governing equations can be expressed in the incompressible unsteady form as follows:

\section{Continuity}

$$
\partial_{x} u+\partial_{y} \mathrm{v}=0
$$

\section{Momentum}

$$
\begin{aligned}
& \partial_{t} u+u \cdot \partial_{x} u+\mathrm{v} \cdot \partial_{y} u=-\partial_{x} p+\operatorname{Pr} . \nabla^{2} u+\operatorname{Pr} . \mathrm{Ha}^{2} \sin \gamma(-u \cdot \sin \gamma+\mathrm{v} \cdot \cos \gamma), \\
& \partial_{t} \mathrm{v}+u \cdot \partial_{x} \mathrm{v}+\mathrm{v} \cdot \partial_{y} \mathrm{v}=-\partial_{y} p+\operatorname{Pr} . \nabla^{2} \mathrm{v}+\operatorname{Pr} \cdot \mathrm{Ha}^{2} \cos \gamma(u \cdot \sin \gamma-\mathrm{v} \cdot \cos \gamma)+\operatorname{Ra} \cdot \operatorname{Pr} . \theta
\end{aligned}
$$

Energy

$$
\partial_{t} \theta+u . \partial_{x} \theta+\mathrm{v} . \partial_{y} \theta=\nabla^{2} \theta
$$


The boundary conditions used in the present study are as follows:

$$
\begin{aligned}
& u(x, 0)=u(x, 1)=u(0, y)=u(1, y)=0, \\
& \mathrm{v}(x, 0)=\mathrm{v}(x, 1)=\mathrm{v}(0, y)=\mathrm{v}(1, y)=0, \\
& \partial_{y} \theta(x, 0)=\partial_{y} \theta(x, 1)=\theta(1, y)=0, \theta(0, y)=1 .
\end{aligned}
$$

The heat transfer is described using the average Nusselt number $\overline{N u}$ along the hot wall:

$$
\overline{N u}=\frac{1}{\Delta T / H} \frac{1}{H} \int_{0}^{H}-\left.\frac{\partial T}{\partial X}\right|_{X=0} d Y=\int_{0}^{l}-\left.\frac{\partial \theta}{\partial x}\right|_{x=0} d y
$$

The convergence criterion for steady state is defined as follows:

$$
\left|\frac{\overline{N u}(t+5000 \Delta t)-\overline{N u}(t)}{\overline{N u}(t)}\right| \leq 10^{-4}
$$

\section{COMPUTATIONAL METHOD}

A BGK lattice Boltzmann is considered in this study for simulating the fluid flow and heat transfer. In the LBM approach, the fluid is modelled by fictitious particle modelled by distribution functions that occupy nodes and transit to neighbouring nodes in a streaming phase. In traditional CFD solvers, the Poisson equation is time consuming and its solution takes typically $80-90 \%$ of the CPU time [15], its absence in LBM means that codes are comparatively fast based on time step per grid point. In our previous works [7,16-20], we have found that a D2Q9-D2Q4 lattice is a suitable model for simulating thermal flows, for its stability compared to the D2Q9-D2Q9 thermal model (see Fig. 2), it preserves the computational efforts, since the collision step takes around $70 \%$ of the CPU time.

The evolution of the distribution functions in the D2Q9-D2Q4 lattice model in the presence of source term $S_{k}$ is written as follows:

$$
\begin{cases}f_{k}\left(\boldsymbol{x}^{\prime}, t^{\prime}\right)-f_{k}(\boldsymbol{x}, t)=-\left(f_{k}(\boldsymbol{x}, t)-f_{k}^{e q}(\boldsymbol{x}, t)\right) / \tau_{v}+\Delta t S_{k}, & k=0-8 \\ g_{k}\left(\boldsymbol{x}^{\prime}, t^{\prime}\right)-g_{k}(\boldsymbol{x}, t)=-\left(g_{k}(\boldsymbol{x}, t)-g_{k}^{e q}(\boldsymbol{x}, t)\right) / \tau_{\alpha}, & k=1-4\end{cases}
$$

Where $\boldsymbol{x}^{\prime}=\boldsymbol{x}+\boldsymbol{e}_{\boldsymbol{k}} \Delta t, t^{\prime}=t+\Delta t$ and the correspondent equilibrium parts $f_{k}^{e q}$ and $g_{k}^{e q}$ are defined as:

$$
\left\{\begin{array}{l}
f_{k}^{e q}(\boldsymbol{x}, t)=\omega_{k} \rho\left(1+3 \boldsymbol{e}_{\boldsymbol{k}} \cdot \boldsymbol{u}+4.5\left(\boldsymbol{e}_{\boldsymbol{k}} \cdot \boldsymbol{u}\right)^{2}-1.5 \boldsymbol{u}^{2}\right) \\
g_{k}^{e q}(\boldsymbol{x}, t)=\varpi_{k} \theta\left(1+2 \boldsymbol{e}_{\boldsymbol{k}} \cdot \boldsymbol{u}\right), \varpi_{k=1-4}=1 / 4
\end{array}\right.
$$

The forcing term $S_{k}$ for incompressible fluid flow is chosen as:

$$
S_{k}=\boldsymbol{F} \cdot \frac{(\boldsymbol{e}-\boldsymbol{u})}{c_{s}^{2}} f_{k}^{e q}
$$

The single-relaxation-times $\tau_{v}$ and $\tau_{\alpha}$ are linked to the kinematic viscosity and the heat diffusivity as:

$$
v=\frac{2 \tau_{v}-1}{6} \frac{\Delta x^{2}}{\Delta t}, \quad \alpha=\frac{2 \tau_{\alpha}-1}{4} \frac{\Delta x^{2}}{\Delta t}
$$


In LB heat and flow modeling philosophy, the macroscopic variables: density, velocity and temperature are computed as:

$$
[\rho(\boldsymbol{x}, t), \rho \boldsymbol{u}(\boldsymbol{x}, t), \theta]=\sum\left[f_{k=0-8}^{e q}, \boldsymbol{e}_{k} \cdot f_{k=0-8}^{e q}, g_{k=1-4}^{e q}\right]
$$

Since it affects the accuracy of the computations, implementation of boundaries conditions is a very important issue in LBM. The second-order bounce back boundary rule for the nonequilibrium distribution function proposed by Zou and He (1997) [21] is used to account for the noslip boundary condition along the four walls as: $\left(f-f^{e q}\right)^{<}=\left(f-f^{e q}\right)^{>}$, where the asterisk "<" and ">" denote for inner (unknown) and outer (known) particles respectively at the wall node:

$$
\begin{aligned}
& f_{2}=\left(f_{4}-f_{4}^{e q}\right)+f_{2}^{e q}, f_{5}=\left(f_{7}-f_{7}^{e q}\right)+f_{5}^{e q} \text { and } f_{6}=\left(f_{8}-f_{8}^{e q}\right)+f_{6}^{e q}: \text { at } y=0, \\
& f_{4}=\left(f_{2}-f_{2}^{e q}\right)+f_{4}^{e q}, f_{7}=\left(f_{5}-f_{5}^{e q}\right)+f_{7}^{e q} \text { and } f_{8}=\left(f_{6}-f_{6}^{e q}\right)+f_{8}^{e q} \text { at } y=1, \\
& f_{1}=\left(f_{3}-f_{3}^{e q}\right)+f_{1}^{e q}, f_{5}=\left(f_{7}-f_{7}^{e q}\right)+f_{5}^{e q} \text { and } f_{8}=\left(f_{6}-f_{6}^{e q}\right)+f_{8}^{e q} \text { at } x=0, \\
& f_{3}=\left(f_{1}-f_{1}^{e q}\right)+f_{3}^{e q}, f_{6}=\left(f_{8}-f_{8}^{e q}\right)+f_{6}^{e q} \text { and } f_{7}=\left(f_{5}-f_{5}^{e q}\right)+f_{7}^{e q} \text { at } x=1,
\end{aligned}
$$

For the temperature field, the temperature distribution functions at the isothermal walls obey to the condition: $g^{<}=-g^{>}+0.5 \theta_{\text {wall }}$. However, the adiabatic boundary condition is transferred to Dirichlet-type condition using the conventional second-order finite difference approximation as: $g_{\text {wall }}=\left(4 g_{\text {wall } \pm \Delta y}-g_{\text {wall } \pm 2 \Delta y}\right) / 3$, which can be summarized as follows:

$$
\begin{aligned}
& g_{1}(0, y)=-g_{3}(0, y)+\left(\varpi_{1}+\varpi_{3}\right) \cdot \theta_{c}: \quad \text { at } x=0, \\
& g_{3}(1, y)=-g_{1}(1, y)+\left(\varpi_{1}+\varpi_{3}\right) \cdot \theta_{h}: \quad \text { at } x=1, \\
& g_{2}(x, 0)=\left[4 g_{2}(x, \Delta y)-g_{2}(x, 2 \Delta y)\right] / 3: \text { at } y=0, \\
& g_{4}(x, 1)=\left[4 g_{4}(x, 1-\Delta y)-g_{4}(x, 1-2 \Delta y)\right] / 3: \text { at } y=1 .
\end{aligned}
$$

\section{ESTABLISHING THE CODE CREDIBILITY}

We have to mention that the grid size sensitivity has been tested previously for the same range of the Rayleigh number $R a$, a uniform grid size $201 \times 201$ will be adopted for the following. To establish the credibility of the thermal lattice Boltzmann developed code, we made two test cases involving different situations of the above mentioned monitoring parameters.

First a square cavity counter-clockwise tilted to $0^{\circ}$ or $20^{\circ}$ from horizontal is considered, the Prandtl number is set to 0.733 and $R a=2 \times 10^{5}$. The streamline traces of the present calculations are plotted in Fig. 3 side-by-side with former numerical and experimental findings reported by Han [13] and Linthorst et al. [22]. Qualitatively, the present streamlines distributions are consistent with the literature results. At the same case, the dimensionless $y$-velocity calculated at the cavity mid-plane for $R a=1.3 \times 10^{5}$ when the enclosure is $0^{\circ}$ or $50^{\circ}$ tilted, are plotted in Fig. 4 . As one can see, the LB method present a good level of predictability compared to the previous experimental results (symbolized in Fig. 4) and numerical predictions of finite volume method using a refined mesh near walls $51 \times 51$ or either using uniform control volumes $201 \times 201$. In the second test case, the present LB predictions are compared to the ADI (Alternating Direction Implicit) and FE methods for natural convection flow in square cavity under external magnetic field effects for different Hartmann numbers. The present LB results are tabulated in Table 1 and gathered with the references findings. As one can remark, our results are in excellent agreement with predictions of conventional approaches. In the following the Rayleigh number $R a$ is set to $10^{5}, H a=0$ or 50 and $P r=0.01,0.1,1$ or 10 and the left hot wall of the cavity is kept parallel to the vertical. 


\section{RESULTS AND DISCUSSIONS}

\section{a) Free buoyant flow $(\mathrm{Ha}=0)$}

The temperature contours and the Streamlines of a fluid flowing for $R a=10^{5}$ without external magnetic field under the effects of varying the Prandtl number are computed. The velocity and Nusselt number local distributions are also plotted.

Streamlines, vorticity, isotherms and heat transfer rate: For $\operatorname{Pr}=0.01$, the flows is unsteady (chaotic) and the dynamic structure changes continually with time, showing two centro-symmetric cells at the core of the cavity; a principle quasi-circle clockwise rotating large cell occupies the enclosure. The flow exhibits also two counter rotating secondary cells at the four corners symmetric by the cavity centre. The isotherms are distorted at the cavity core and more stretched and piled near the cavity mid-height. It is worth-noting that this type-flow (low Prandtl number flows) characterizing liquid metal show usually time dependent behaviors and instabilities.

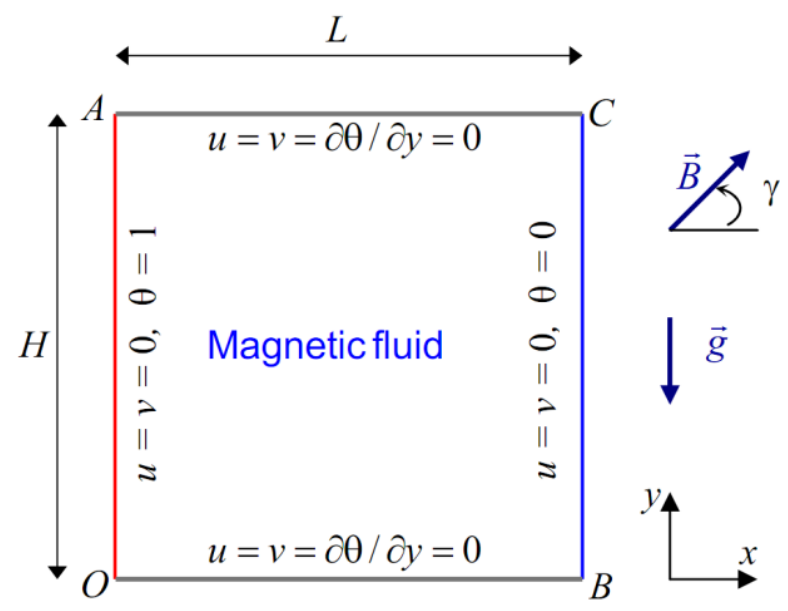

Figure 1: Configuration of the physical and computational model.

Table 1: Comparison of the calculated Nusselt number with referenced former works using different methods.

\begin{tabular}{ccccc}
\hline$R a / P r$ & $H a$ & ADI [23] & FEM [24] & LBM \\
\hline $2 \times 10^{4}$ & 0 & 2.5188 & 2.5439 & 2.5516 \\
& 10 & 2.2234 & 2.2385 & 2.2458 \\
& 100 & 1.0110 & 1.0066 & 1.0128 \\
\hline $2 \times 10^{5}$ & 0 & 4.9198 & 5.0245 & 5.1276 \\
& 10 & 4.8053 & 4.9136 & 4.9252 \\
& 100 & 1.4317 & 1.4292 & 1.4467 \\
\hline
\end{tabular}




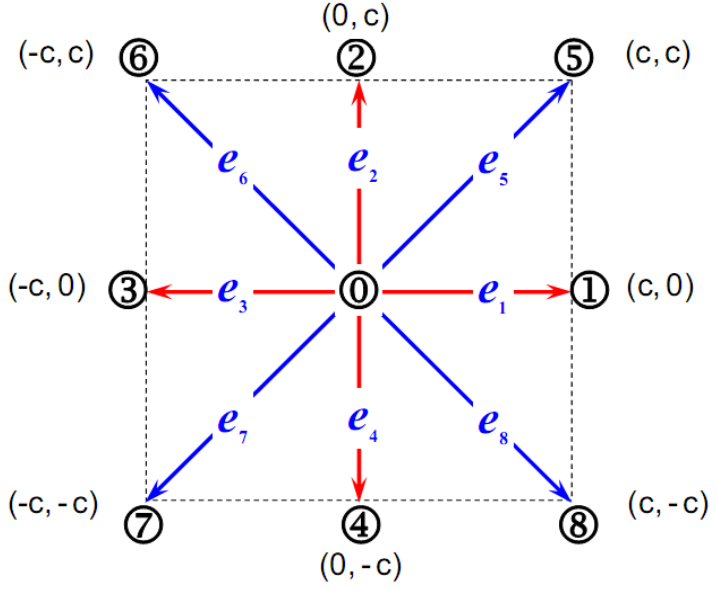

Figure 2: The nine-particle speeds used in the hydrodynamic of lattice Boltzmann equation. Only the four red velocities are necessary for the temperature field.

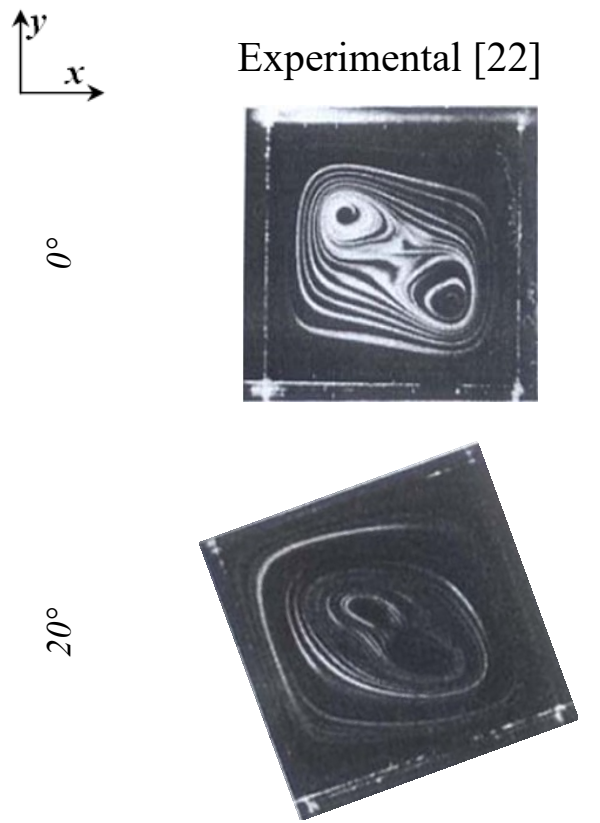

Finite Volume [13]

Present LBM
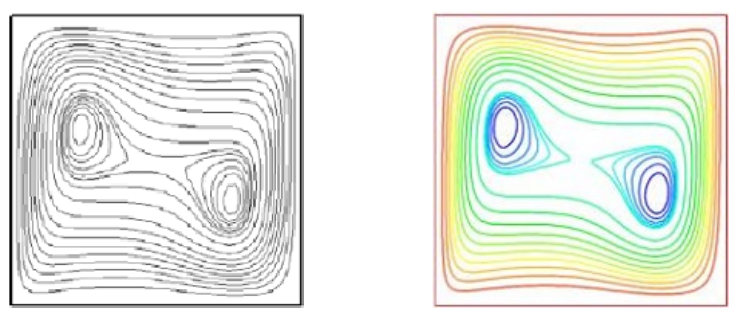

Figure 3: Comparison of present streamlines calculation with former experimental and numerical findings for $P r=0.733, R a=2 \times 10^{5}$ and different cavity inclinations. 


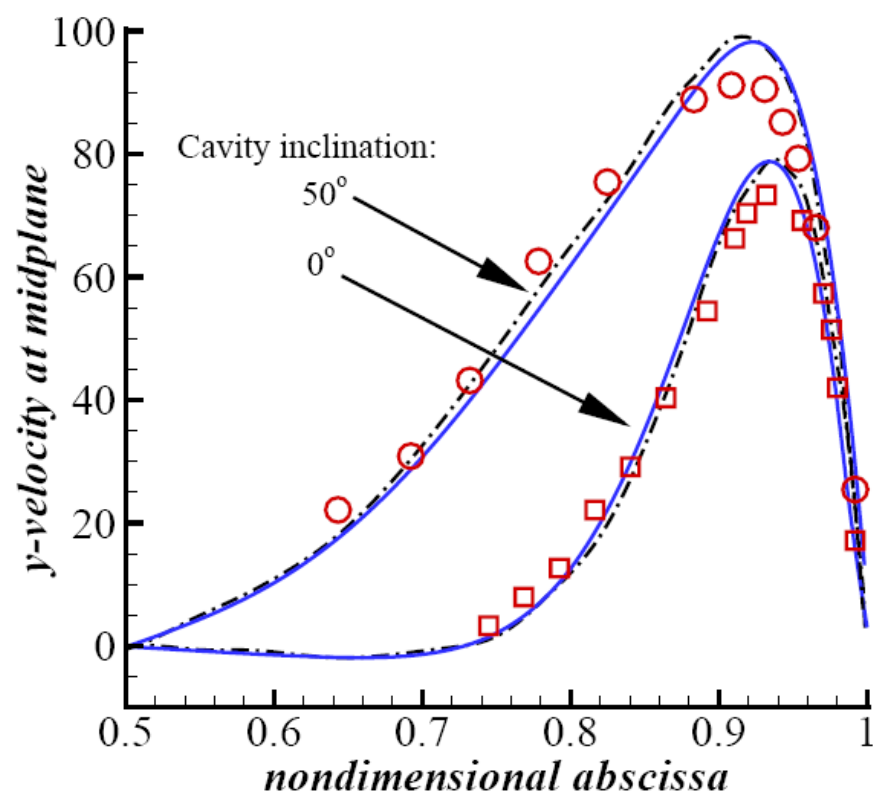

Figure 4: Comparison of dimensionless $y$-velocities at cavity mid-plane for $\operatorname{Pr}=0.733$, $R a=1.3 \times 10^{5}$ and different cavity inclinations. Symbols: experimental results of [22], dashdotted lines: FVM results of [13] and blue-solid lines: present predictions.

The Nusselt number time history is gathered to its spectra of amplitude frequency in Figure 5, the spectra exhibit four principal oscillating frequency close to $0.9,36.6,37.5$ and 38.4. The timeaverage Nusselt number is close to 3.19. Increasing the Prandtl number to 0.1 the flows behaviour changes distinctly (see Fig. 6): a thermal stratification appears and the dynamic structure shows only three counter rotating cells, and the flow is also steady. The Nusselt number is close to 3.93 and the absolute maximum stream-function is 7.84. Increasing more the Pr number to 1 and 10, the flow exhibits only two centro-symmetric rotating cells at the cavity core. For $\operatorname{Pr}=1$, the left cell centre is the highest, however it is the lower for $P r=10$ and the cells are smaller compared to the $P r=1$ case. This is certainly due to the fact that a rise in the Pr number leads to a rise in viscosity (against the diffusivity of the fluid) which requires the sufficient buoyancy force to conquer viscous forces. The isotherms are more stretched to the isothermal walls and the thermal stratification is intensified. The Nusselt numbers $\overline{N u}$ are 4.60 and 4.72 and the maximum stream-function magnitudes $|\psi|_{\max }$ are 10.02 and 11.11 for respectively $\operatorname{Pr}=1$ and $\operatorname{Pr}=10$. However, the maximum vorticity magnitude is close to 2333, 2428 and 2584 for respectively $P r=0.1,1$ and 10 .
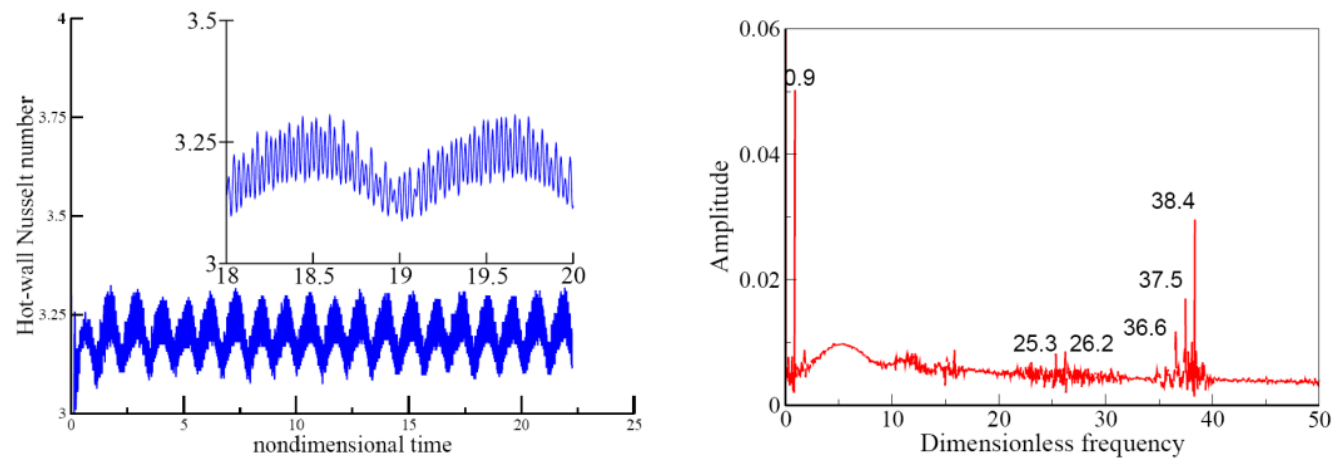

Figure 5: Nusselt time-history (left) and its frequency spectrum (right) for $R a=10^{5}, P r=0.01$ and $H a=0$. 

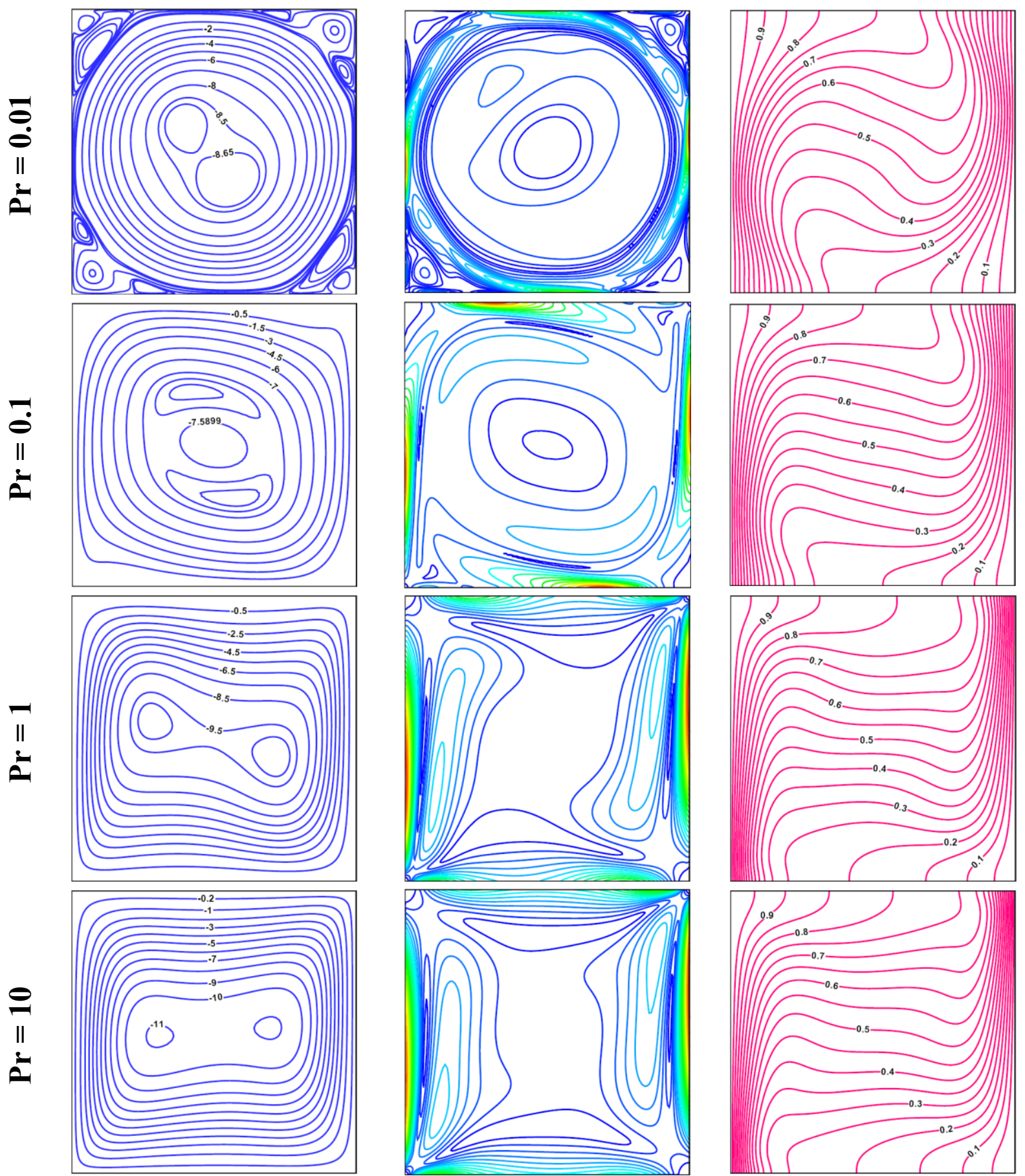

Figure 6: Streamlines, vorticity magnitude and isotherms plots for $R a=10^{5}, H a=0$ and different Prandtl numbers.

Velocity and Nusselt number local distributions: Local fields' distributions can also help to comprehend the flow behaviour near particular zones. In Fig. 7 are plotted the profiles of local distributions of the Nusselt number and velocities at mid planes $x=0.5$ and $y=0.5$ for different Prandtl numbers. As one can see, for low Pr, oscillations appear for the velocity components and the Nusselt number. However for high Pr, the y-velocity acts only near isotherms walls, its nullity from $x=0.3$ to $x=0.7$ clearly explains the thermal stratification formed parallel to $x$-axis as mentioned here-above. Besides, the three fields' profiles show that the behaviour is independent of the $\operatorname{Pr}$ for high values ( $\operatorname{Pr}=1$ and 10) which has been emphasized previously. 

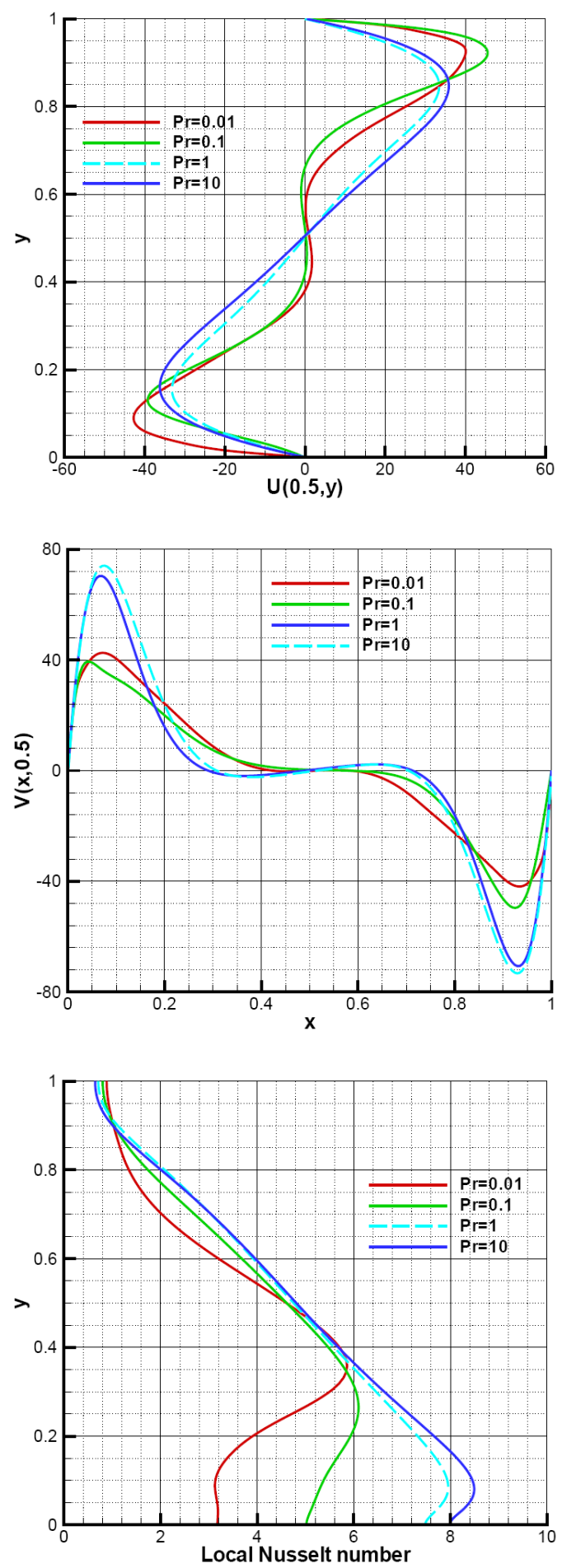

Figure 7: Local distributions of the velocity components at mid-planes $u(0.5, y)$ and $v\left((x, 0.5)\right.$ and the hot-wall Nusselt number for different $P r, R a=10^{5}$ and $H a=0$.

\section{b) Fluid flow under horizontal magnetic field $\left(\gamma=0^{\circ}\right)$}

Streamlines, vorticity, isotherms and heat transfer rate: A simple scaling procedure leads to the following form of the non-dimensional Lorentz force $\boldsymbol{F}_{l} \equiv \mathrm{Pr}_{\mathrm{Ha}}{ }^{2}[-\sin \gamma(u \cdot \sin \gamma-v \cdot \cos \gamma), \cos \gamma$ $(u \cdot \sin \gamma-v \cdot \cos \gamma)]$ and the buoyancy force $\boldsymbol{G}=\operatorname{Ra} \cdot \operatorname{Pr} . \theta \cdot \boldsymbol{e}_{y}$. When the magnetic field is applied parallel to the $\mathrm{x}$-axis (case $\gamma=0^{\circ}$ ) the Lorentz force reduces to $\boldsymbol{F}_{l}=-\mathrm{Pr} \cdot \mathrm{Ha}^{2} \boldsymbol{v}$. Accordingly, the magnitude of ratio buoyancy force by the Lorentz force is proportional to $R a / H a^{2}$, so that it acts directly against the buoyancy force without overcoming it. 
Furthermore, one knows that the Lorentz force reduces velocities and dumps the convection currents and consequently reduces the heat transfer. As a result, major effects are observed between the cases: with/without applying magnetic field (see Fig. 8): the dynamic and thermal structures of the flow for $\mathrm{Ha}=0$ change entirely for a moderate magnetic field magnitude of $\mathrm{Ha}=50$; a likeelongated core-cell pattern is remarked for $\operatorname{Pr} \geq 0.1$ and a weak thermal stratification along the first diagonal of the cavity occurs and is kept unchanged under the Prandtl number increase.
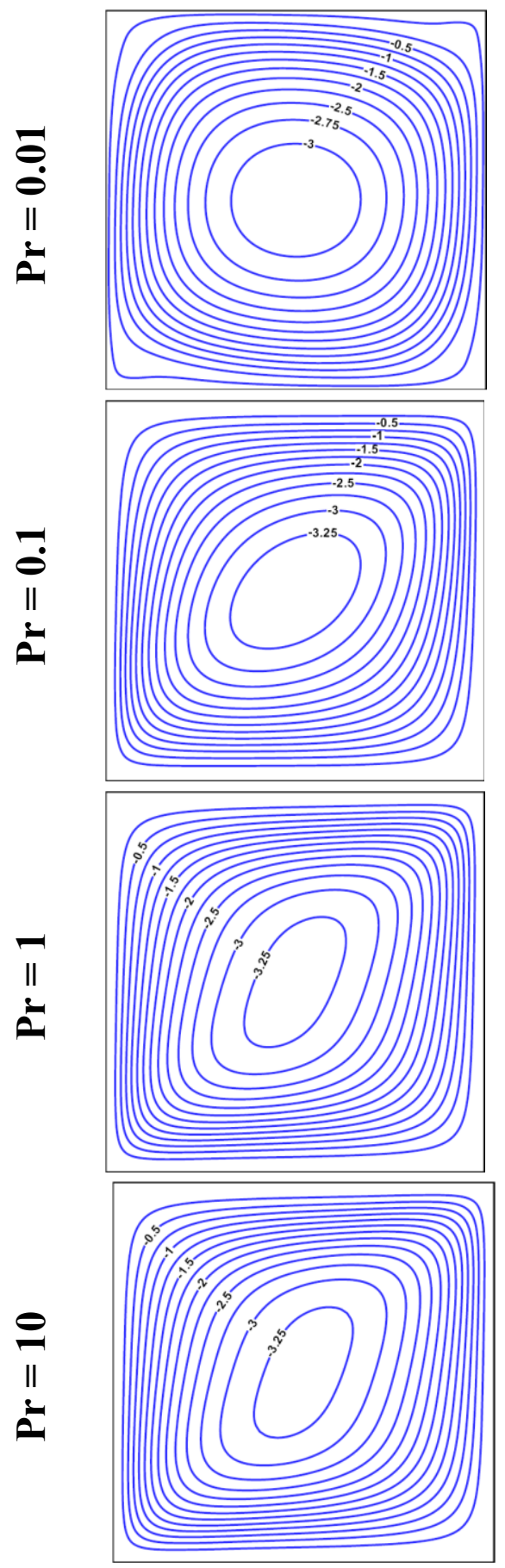
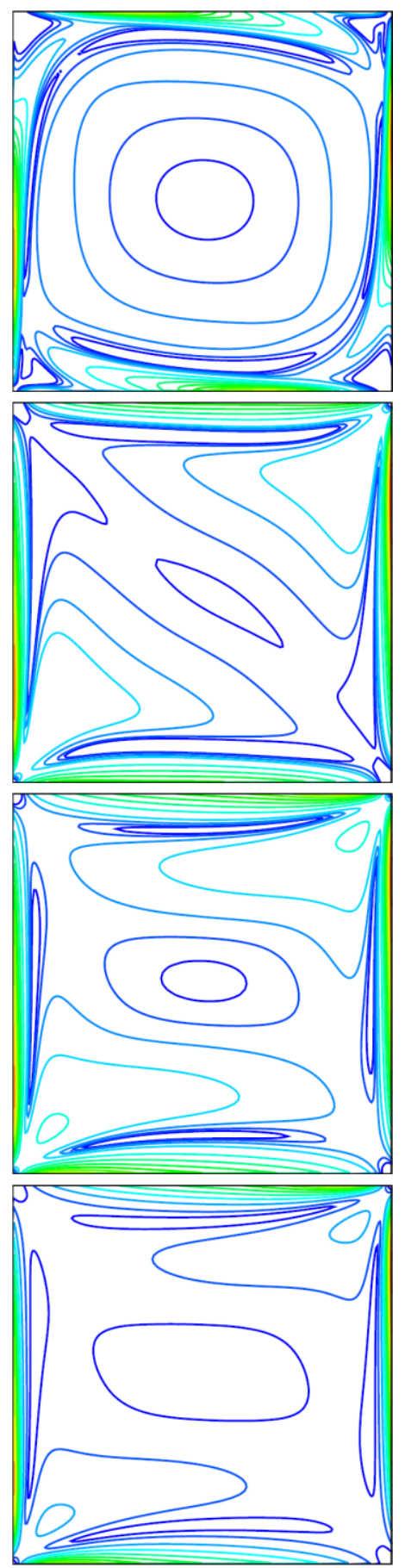
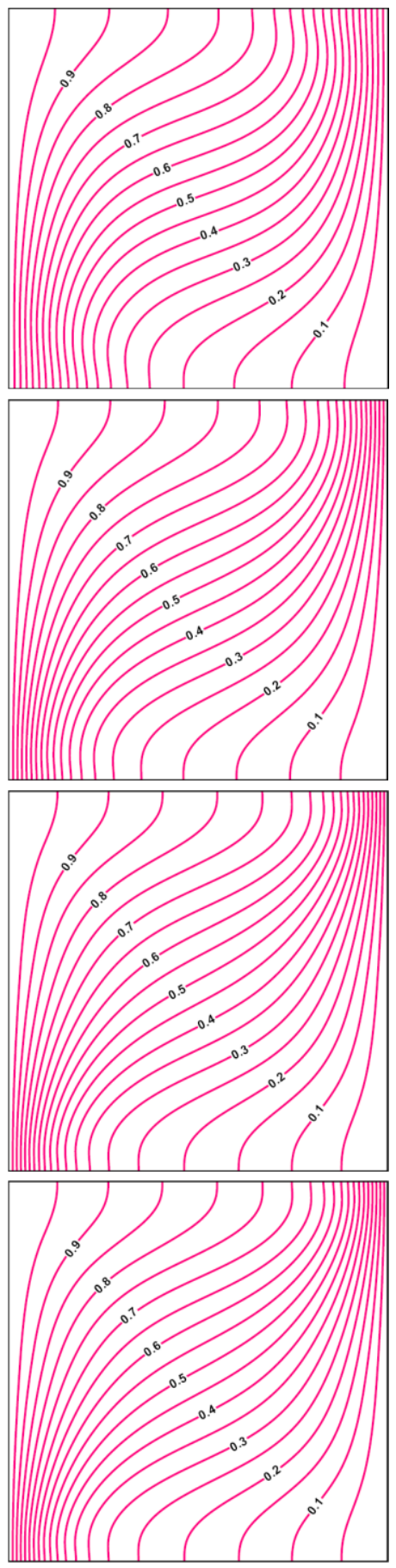

Figure 8: Streamlines, vorticity magnitude and isotherms plots for $R a=10^{5}, H a=50, \gamma=0^{\circ}$ and different Prandtl numbers.

One can also remark that the thermal boundary layers at the two side walls tend to disappear so that the Nusselt number is decreased to half compared to $H a=0$ case as will be given in the following and $|\psi|_{\max }$ diminishes at least of the third party. 
The stream-function magnitude does not changed much, for $\operatorname{Pr}=0.01,0.1,1$ and 10 we have $|\psi|_{\max }=3.22,3.48,3.36$ and 3.35 respectively, then a maximal change amount of $8.1 \%$. The same conclusion applies for the heat transfer, the Nusselt number is 2.03, 2.15, 2.16 and 2.17 when increasing the Prandtl number, then a maximal change amount of $15.6 \%$ to reference value $\overline{N u}=2.03$. We should mention that the lower Nusselt number $\overline{N u}=2.03$ is superior to unit then the convective currents still act, this is due to the fact that the ratio $R a / H a^{2}=10^{5} / 50^{2}=40$ which is so greater than unit. The maximum vorticity magnitude is close to $1124,1136,1205$ and 1323.

Velocity and Nusselt number local distributions: The plots of the velocity components at mid-planes and the local Nusselt number along the hot wall are presented in Fig. 9. Compared to Fig. 7, one can say that the maximum velocities have decreased significantly to almost the quarter due to the magnetic force dumping. The hot-wall local Nusselt number maximum is near the bottom wall because of the cooled right wall. It is also observed that there is no significant effect on the heat transfer rate when $\operatorname{Pr} \geq 1$.

\section{c) Fluid flow under vertical magnetic field $\left(\gamma=90^{\circ}\right)$}

Streamlines, vorticity, isotherms and heat transfer rate: The predicted results are shown in Fig. 10. In this case of $\gamma=90^{\circ}$, the Lorentz force reduces to $\boldsymbol{F}_{l}=-\operatorname{Pr} . \mathrm{Ha}^{2} \boldsymbol{u}$ and consequently it acts counter the horizontal flow currents. Effectively, for low Prandtl number $(P r=0.01)$ the core-cell is extended according to the second diagonal with two small cells at the correspondent corners. However, by increasing the Prandtl number (ie increasing the viscous force) the viscous force acts according to "- $y$ "; the resulting force leads to a two cells elongated according to the first diagonal, with alteration rising with increasing the Prandtl number to 10.

The Prandtl number effect, as one can see, is more expressed on dynamic structure more than the thermal structure. The isotherms exhibit a stretching pattern along the first diagonal with slight clockwise rotation under increasing $\mathrm{Pr}$; this behaviour is more expressed compared to the case of horizontal magnetic field, but examining the flow pattern under increasing the Prandtl number at vertical magnetic field we can state that $\operatorname{Pr}$ has a minor effect at high values, this conclusion remains valid either for the case without magnetic field. The behaviour for respectively $P r=0.01$, $0.1,1$ and 10, is the same for the stream-function magnitude $|\psi|_{\max }$ which is close 3.09, 3.42, 3.54 and 3.54 and the hot-wall Nusselt number to 2.05, 2.32, 2.37 and 2.37 then a decreased heat transfer rate. However, the maximum vorticity magnitude is close to $1408,1356,1370$ and 1409. A thermal gradient is developed parallel to the first diagonal, rising under the Prandtl number increase. The thermal boundary layers at the vertical sides disappear leading to decrease in the heat transfer rate quantified by the Nusselt number.

Velocity and Nusselt number local distributions: The simulated profiles are depicted in Fig. 11. For $\operatorname{Pr} \geq 0.1$ and for a vertical magnetic field, the resulting magnetic force suppresses the horizontal convective currents and as a results the peaks for $u(0.5, y)$ are the weaker compared to previous cases. However for the $y$-velocity, peaks are a little high due to absence of magnetic removal. The general behaviour shows that no significant changes observed on both local velocity and Nusselt number by increasing $P r$. This is in agreement with the computed streamfunction magnitudes and the average Nusselt numbers which are very close. Contrarily, for $P r=0.01$, the profiles' patterns differ slightly because of the unsteadiness appearing for low Prandtl numbers. 

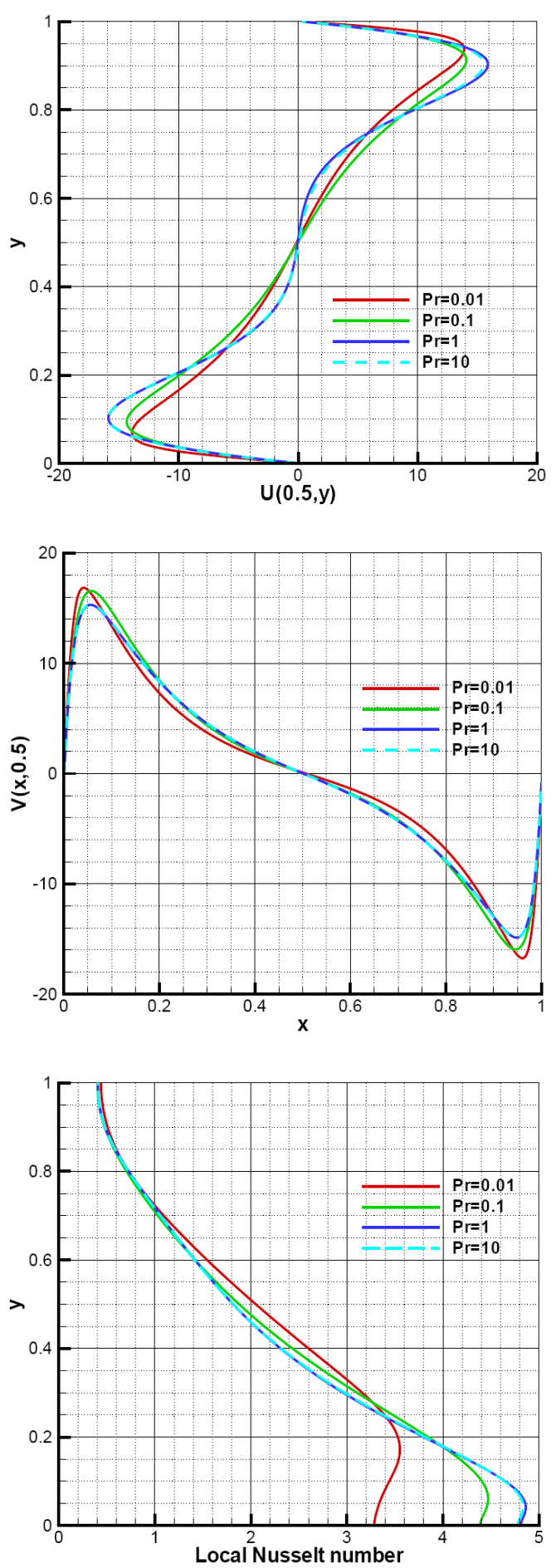

Figure 9: Local distributions of the velocity components at mid-planes $u(0.5, y)$ and $v(x, 0.5)$ and the hot-wall Nusselt number for different $\operatorname{Pr}, \mathrm{Ra}=10^{5}, \mathrm{Ha}=50$ and $\gamma=0^{\circ}$.

\section{CONCLUSION}

We consider in present study an unsteady, two-dimensional MHD natural convection within electrically conducting filled square enclosure in the presence of inclined magnetic field for different Prandtl number. The governing equations are solved using the lattice Boltzmann method for the differentially heated cavity problem. It was found that for low Prandtl number flow without magnetic field, the cavity hot-wall Nusselt number exhibited time dependent behavior and the flow is multicellular. However, by increasing the Prandtl number, a transition to bicellular flow occurs. 

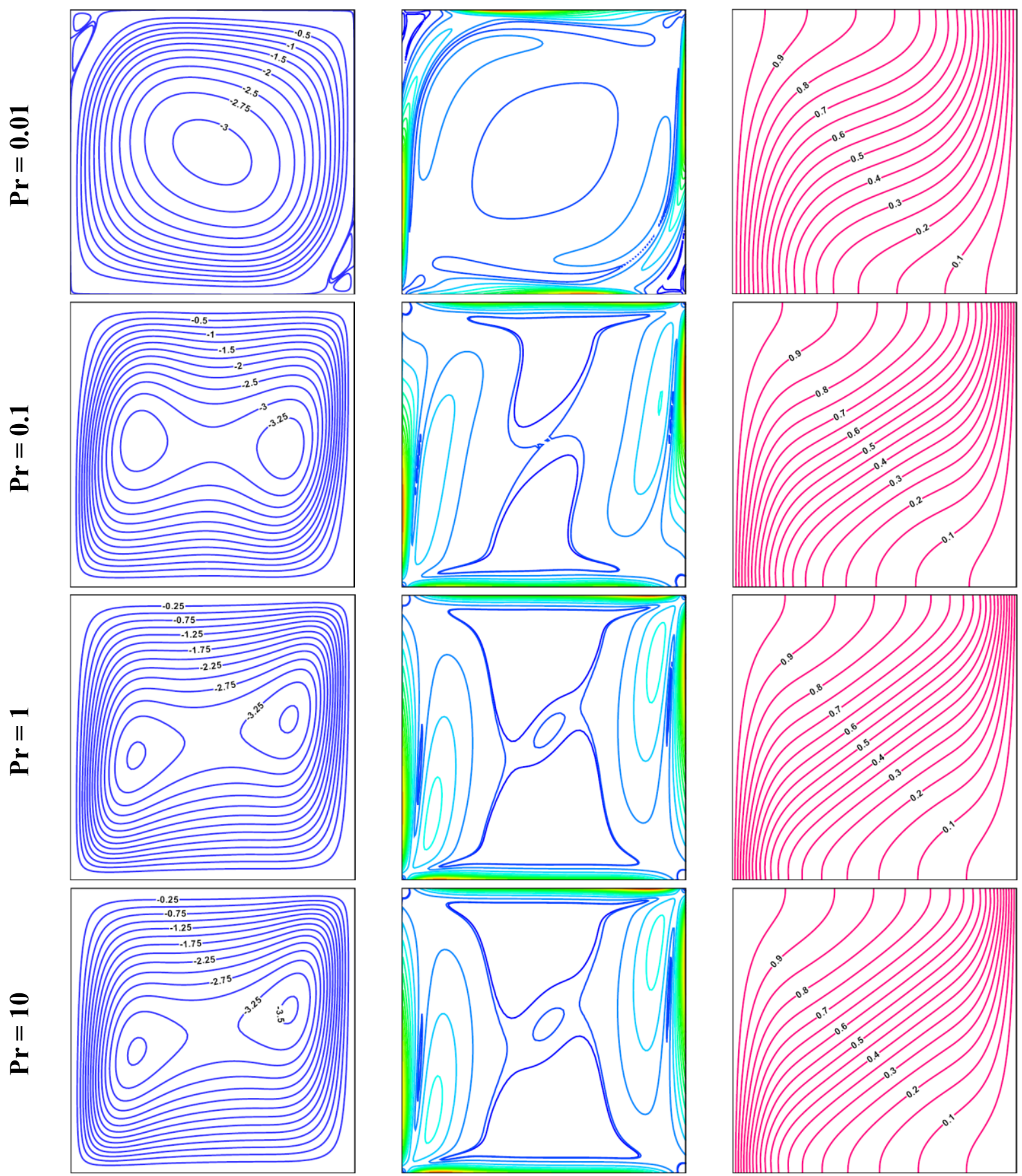

Figure 10: Streamlines, vorticity magnitude and isotherms plots for $\mathrm{Ra}=10^{5}, \mathrm{Ha}=50, \gamma=90^{\circ}$ and different Prandtl numbers.

Besides, by applying the external magnetic field at low Prandtl number, the convective heat transfer rate is reduced considerably and no unsteady state are observed in the tested monitoring parameters ranges. Furthermore, by increasing the Prandtl number, the flow is always bicellular and the coupled effect of the Lorentz force and viscous force results in dumping the convective currents so that no great changes are observed between $P r=1$ and 10 on isotherms and heat transfer quantified using the Nusselt number, however the signature (of varying $P r$ ) is well clear on the streamlines. 

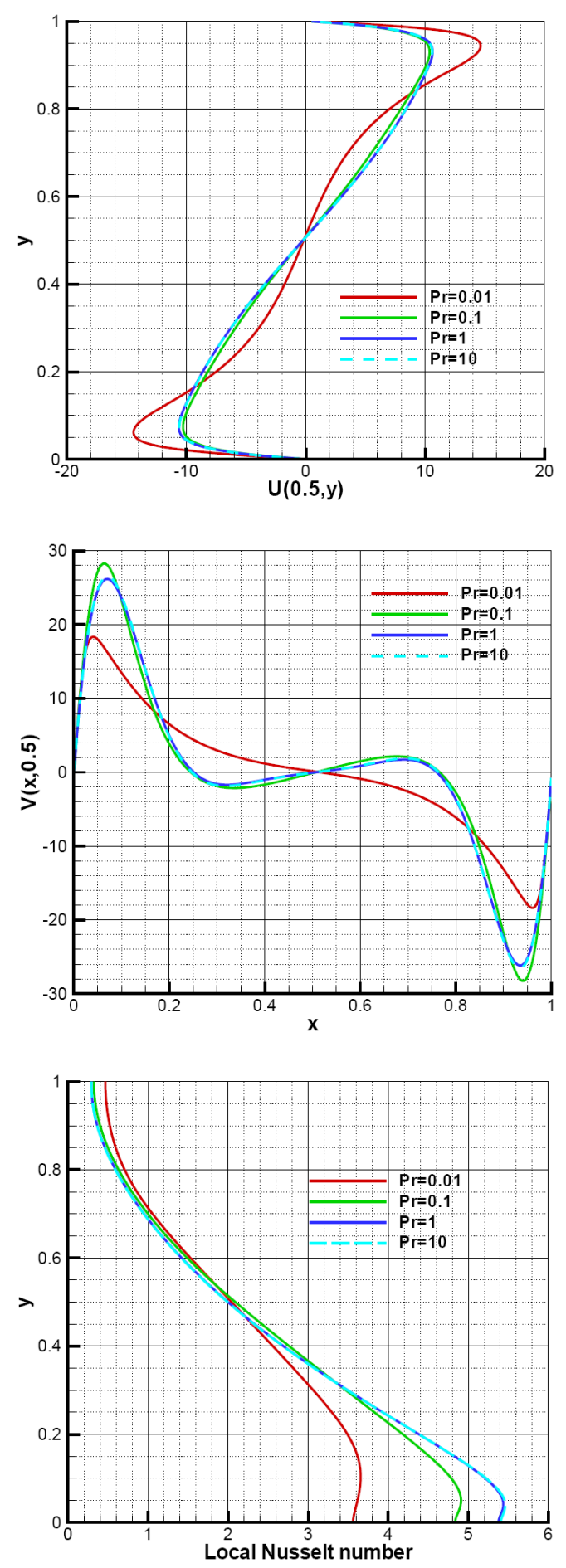

Figure 11: Local distributions of the velocity components at mid-planes $u(0.5, y)$ and $v(x, 0.5)$ and the hot-wall Nusselt number for different $\mathrm{Pr}, \mathrm{Ra}=10^{5}, \mathrm{Ha}=50$ and $\gamma=90^{\circ}$.

It is found also, through this study, that the LB method is a promising tool for investigating MHD convective heat transfer in confined space. Moreover, the LB approach allows more simply accounting for complex physics such as external magnetic force and particularly preserves the computational cost. Besides, regarding the CPU time, we noted that the in presence of the magnetic force, the flow takes extremely long time to reach steady state.

Beyond the low-Prandtl number flow applications as listed in the introduction section, the magneto-convection studies serve for some others quite promising applications related to metallurgy, such as magnetic-levitation casting and MHD stirring of molten metal. This computational study falls into these categories and is intended to give a solution of compute intensive engineering problems and presents a prerequisite for the evolution of the e-science. 
In our future works we will focus on the effects of the presented monitoring parameters on the occurrence of bifurcation phenomena inside confined spaces. This issue is met with continued interest by researchers [25].

\section{Acknowledgement}

The first author gratefully acknowledges Prof. Z. Guo (Huazhong University of Science and Technology, Wuhan, China) for fruitful discussions. He, also, thanks the Ministry of Higher Education and Scientific Research -Tunisia, for the financial support and particularly the University of Limoges for the computational facilities provided by CALI calculation centre.

\section{References}

[1] Gelfgat, A. Y. and P.-Z. Bar-Yoseph, The effect of an external magnetic field on oscillatory instability of convective flows in a rectangular cavity. Physic of Fluids, 2001. 13(8), p. 2269-2278.

[2] Roussellet, V., X. Niu, H. Yamaguchi and F. Magoulés, Natural convection of temperaturesensitive magnetic fluids in porous media. Advances in Applied Mathematics and Mechanics, 2011. 3(1), p. 121-130.

[3] Hadavand, M. and A.C.M. Sousa, Simulation of thermomagnetic convection in a cavity using the lattice Boltzmann model, Journal of Applied Mathematics. 2011. Article ID 538637, 14 pages; doi:10.1155/2011/538637.

[4] Ece, M.C. and Büyük E. (2006): Natural-convection flow under a magnetic field in an inclined rectangular enclosure heated and cooled on adjacent walls. Fluid Dynamics Research, vol. 38, pp. 564-590.

[5] Hasanpour, A., Farhadi, M., K. Sedighi and H. R. Ashorynejad, Numerical Study of Prandtl Effect on MHD Flow at a Lid-Driven Porous Cavity. International Journal for Numerical Methods in Fluids, 2012. 70(7), p. 886-898.

[6] Jina, L. and X.-R. Zhang, Analysis of temperature-sensitive magnetic fluids in a porous square cavity depending on different porosity and Darcy number. Applied Thermal Engineering, 2013. 50(1), p. 1-11.

[7] Bao J., Schaefer L., Lattice Boltzmann equation model for multi-component multi-phase flow with high density ratios, Applied Mathematical Modelling, 37(4), 2013, p. 1860-1871.

[8] Chieh-Li C., Shing-Cheng C., Cha'o-Kuang C., Chun-Kai C., Lattice Boltzmann simulation for mixed convection of nanofluids in a square enclosure, Applied Mathematical Modelling, 39(8), 2015, p. 2436-2451.

[9] Taghilou M., Rahimian M. H., Lattice Boltzmann model for thermal behavior of a droplet on the solid surface, International Journal of Thermal Sciences, 86, 2014, p. 1-11.

[10] R. Djebali, ElGanaoui, M., Jaouabi A., Pateyron B.; Lattice Boltzmann scrutiny of spray jet and impact characteristics under dispersion effects of powder injection parameters in APS process; International Journal of Thermal Sciences, vol. 100, pp. 229-239, 2016.

[11] Hao, L., Xinhua, L. and Yongzhi, L., The lattice Boltzmann simulation of magnetic fluid, Procedia Engineering, 2011. 15, p. 3948-3953.

[12] Chatterjee, D. and S. Amiroudine, Lattice Boltzmann simulation of thermofluidic transport phenomena in a DC magnetohydrodynamic (MHD) micropump. Biomed Microdevices, 2011. 13, p. 147-157.

[13] Han, C. Y., Effect of a magnetic field on natural convection of an electrically conducting fluid in a tilted cavity. Journal of the Korean Physical Society, 2009. 55(5), p. 2193-2199. 
[14] Zhang, X.-R., L.-C.Jin, X.-D. Niu, and H. Yamaguchi, Lattice Boltzmann simulation for magnetic fluids in porous medium. Physics Procedia, 2010. 9, p. 162-166.

[15] Madabhushi, R.K. and S.P. Vanka, Large eddy simulation of turbulence driven secondary flow in a square duct. Physic of Fluids A, 1991. 3, p. 2734-2745.

[16] Djebali, R., B. Pateyron, and M. El Ganaoui, A lattice Boltzmann-based study of plasma sprayed particles behaviours. CMC: Computers, Materials \& Continua, 2011. 25(2), p. 159176.

[17] Djebali, R. and M. El Ganaoui, Assessment and computational improvement of thermal lattice Boltzmann models based benchmark computations. CMES: Computer Modeling in Engineering \& Sciences, 2011.71 (3), p. 179-202.

[18] Djebali, R., M. El Ganaoui, R. Pateyron, and H. Sammouda, Simulation and modeling of turbulent plasma jet based on axisymetric LBGK model. Defect and Diffusion Forum, 2011. vols. 312-315, p. 1167-1171.

[19] Djebali, R. , M. El Ganaoui and B. Pateyron, A lattice Boltzmann based investigation of powder in-flight characteristics during APS process, Part I: modelling and validation. Progress in Computational Fluid Dynamics: an int. Journal, 2012. 12(4), p. 270-278.

[20] El Ganaoui, M. and R. Djebali, Aptitude of a lattice Boltzmann method for evaluating transitional thresholds for low Prandtl number flows in enclosures, Compte Rendu Mecanique, 2010. 338, p. 85-96.

[21] Zou, Q. and X. He, On pressure and velocity boundary conditions for the lattice Boltzmann BGK model. Physics of Fluids, 1997. 9(6), p. 1591-1598.

[22] Linthorst, S. J., M. W. M. M. Schinkel and C. J. Hoogendoorn, Flow structure with natural convection in inclined air-filled enclosures. ASME J. Heat Transfer, 1981. 103(3), p. 535539.

[23] Rudraiah, N., R. M. Barron, M. Venkatachalappa and C.K. Subbaray, Effect of a magnetic field on free convection in a rectangular enclosure, International Journal of Engineering Sciences, 1995. 33(8), p. 1075-1084.

[24] Sathiyamoorthy, M. and A. J. Chamkha, Natural convection flow under magnetic field in a square cavity for uniformly (or) linearly heated adjacent walls. Int. J. of Numerical Methods for Heat \& Fluid Flow, 2012. 22(5), p. 677-698.

[25] Hammami F., Ben Cheikh N., Ben Beya B., On the Importance of the Influence of both Velocity and Aspect Ratios on the Occurrence of Bifurcation Phenomena within a TwoSided Lid-Driven Enclosure, International Letters of Chemistry, Physics and Astronomy, Vol. 55, pp. 160-172, 2015 